\title{
Sea-Trial Results for Cyclic-Prefix OFDM with Long Symbol Duration
}

\author{
Christian R. Berger*, João Gomes ${ }^{\dagger}$, and José M. F. Moura* \\ *Dept. of Electrical \& Computer Engineering, Carnegie Mellon University, Pittsburgh, PA 15213, USA \\ $\dagger$ Institute for Systems and Robotics, Instituto Superior Técnico, 1049-001 Lisboa, Portugal
}

\begin{abstract}
In this paper we report results from the Calibration and Communications Sea Trial 2010. We explain in detail the used cyclic-prefix orthogonal frequency division multiplexing (OFDM) signal design and adopted receiver processing, based on sparse channel estimation and intercarrier interference (ICI) equalization. We then focus on two phenomena that are not commonly treated in wireless communications literature, namely, colored noise due to (partially) untreated ICI and narrowband interference, and biased symbol estimates due to noisy channel estimates used in multi-phone combining.
\end{abstract}

Index Terms-Underwater acoustic, multicarrier transmission, sparse channel estimation, colored noise, biased symbol estimates.

\section{INTRODUCTION}

Underwater acoustic (UWA) channels feature long delay spreads and significant Doppler effects, due to internal waves, platform and sea-surface motion [1]. Besides the established singlecarrier approach [2], multicarrier transmission has been investigated intensely in recent years, see e.g. [3]-[11]. A definitive advantage of multicarrier transmission is that the complexity of channel equalization in the frequency domain is independent of the channel delay spread. The caveat is that the symbol length in multicarrier transmission needs to be significantly longer than the delay spread to avoid loss of spectral efficiency due to the overhead caused by the guard interval or cyclic-prefix (CP). A long symbol duration in turn makes multicarrier transmission more sensitive to Doppler effects that cause intercarrier interference (ICI).

While there are some channels where this tradeoff can be settled satisfactorily, these are mostly reliant on good weather that leads to low wind and wave activity [3]-[7]. It seems therefore pertinent to consider ICI as an integral part of multicarrier transmission on UWA channels [8]-[11] that needs to be estimated and mitigated. In this light, considering long symbol durations that increase spectral efficiency is an attractive option.

In this paper, we report results from a data set collected during the Calibration and Communications Sea Trial 2010 (CalCom'10), which was conducted by CINTAL, Universidade do Algarve from June 21-25, off the coast of Portugal, approximately $30 \mathrm{~km}$ south of the port of Vilamoura. Specifically, we describe in detail the used CP orthogonal frequency division multiplexing (OFDM) signal design with long symbol duration, including coding, modulation, and peakto-average power ratio (PAPR) control. We also cover the considered receiver processing, consisting of demodulation, sparse channel estimation, multi-phone combining, ICI equalization, and soft-input error correction. Sparse channel estimation has been recently shown to be a highly attractive option in UWA communications [5], [10], [12].

After outlining the signal design and receiver processing, we find two unexpected effects in the experimental results that warrant further treatment:

1) The decoding performance suffers significantly due to the colored noise spectrum caused by untreated ICI in general and a narrowband interference source present only at the tenth receive element of the receiver array.

2) Symbol estimates are biased, which can be modeled as a constant amplitude scaling; this leads to increased demodulation errors for higher order constellations like quadrature amplitude modulation (QAM).

We address the colored noise issue by estimating the noise power spectral density (PSD) based on null subcarriers and interpolation as in [11]. Then this information can be used to correctly weight subcarriers in multi-phone combining according to their reliability, where the noise PSD corresponds to the noise level on each subcarrier. Differently from [11], we do not use a structured model of the noise PSD, but simply assume that the PSD is smooth in logarithmic scale. This is sufficient to remedy the detrimental effect of the narrowband interference, but more sophisticated treatment might lead to improved performance.

We investigate the bias of symbol estimates, which varies with the considered channel estimation scheme. While symbol estimates are biased towards zero, or scaled down, when using a conventional least-squares (LS) estimator or orthogonal matching pursuit (OMP) [13], they tend to be too large in magnitude when considering basis pursuit (BP) [14]; see the cited work on sparse channel estimation for more details on OMP/BP algorithms. We discuss more details of our findings and offer a simple remedy that scales up LS and OMP symbol estimates based on assumed channel estimation errors.

The rest of this paper is organized as follows, in Section II we describe the signal design, in Section III we explain the used peak-to-average power control, in Section IV we review the adopted channel model, in Section V we detail the receiver processing, in Section VI we estimate the colored noise PSD, in Section VII we investigate the biased symbol estimates, and finally we conclude in Section VIII. 
TABLE I

OFDM SIGNAL SPECIFICATIONS FOR CALCOM'10 EXPERIMENT.

\begin{tabular}{llr}
\hline symbol length & $T$ & $491.52 \mathrm{~ms}$ \\
cyclic-prefix & $T_{\mathrm{cp}}$ & $48 \mathrm{~ms}$ \\
block length & $T^{\prime}$ & $539.52 \mathrm{~ms}$ \\
\hline no. subcarriers & $K$ & 4096 \\
subcarrier spacing & $1 / T$ & $2.0345 \mathrm{~Hz}$ \\
baseband sampling rate & $B$ & $8.33 \mathrm{kHz}$ \\
carrier frequency & $f_{c}$ & $12 \mathrm{kHz}$ \\
\hline band protection & $N_{\mathrm{P}}$ & 128 \\
effective bandwidth & $B_{\mathrm{eff}}$ & $7.8125 \mathrm{kHz}$ \\
\hline blocks per frame & $N_{\mathrm{B}}$ & 10 \\
\hline
\end{tabular}

\section{Signal Design}

We consider cyclic-prefix (CP) orthogonal frequency division multiplexing (OFDM); let $T$ denote the OFDM symbol duration and $T_{\mathrm{CP}}$ the length of the $\mathrm{CP}$. The total duration of one OFDM block is $T^{\prime}=T+T_{\mathrm{CP}}$ and the subcarrier spacing is $1 / T$. The $k$ th subcarrier is at frequency

$$
f_{k}=f_{c}+k / T, \quad k=-K / 2, \ldots, K / 2-1,
$$

where $f_{c}$ is the carrier frequency and $K$ subcarriers are used, so that the nominal bandwidth is $B=K / T$. Let $s[k]$ denote the information symbol to be transmitted on the $k$ th subcarrier, chosen from a constellation like quadrature phase shift keying (QPSK) or quadrature amplitude modulation (QAM). The nonoverlapping sets of active subcarriers $\mathcal{S}_{A}$ and null subcarriers $\mathcal{S}_{N}$ satisfy $\mathcal{S}_{A} \cup \mathcal{S}_{N}=\{-K / 2, \ldots, K / 2-1\}$; the null subcarriers are used to facilitate Doppler compensation at the receiver [4], and protect the band edges. A transmitted OFDM block in passband is given by

$$
\tilde{x}(t)=2 \operatorname{Re}\left\{\left[\sum_{k \in \mathcal{S}_{\mathrm{A}}} s[k] e^{j 2 \pi \frac{k}{T} t}\right] e^{j 2 \pi f_{c} t}\right\}, t \in\left[-T_{\mathrm{CP}}, T\right] .
$$

A burst of $N_{\mathrm{B}}$ OFDM blocks is transmitted at a time, each carrying independent information symbols, and shifted by $T^{\prime}$.

The parameters used in the CalCom' 10 experiment are listed in Table I; the main design considerations are:

- the CP needs to be longer than the largest expected channel delay spread of about $40 \mathrm{~ms}$;

- the symbol duration should be large relative to the $\mathrm{CP}$ length to improve spectral efficiency; and

- the signal needs to be well contained in the intended frequency band $8-16 \mathrm{kHz}$.

The first point leads to a $\mathrm{CP}$ length of $48 \mathrm{~ms}$, which leaves some margin for timing errors. The OFDM block is chosen as approximately ten times the $\mathrm{CP}$ length to minimize the overhead introduced by the CP. Finally $N_{\mathrm{P}}=128$ null subcarriers are placed on each band edge to strictly limit the transmit spectrum between $8-16 \mathrm{kHz}$. This reduces the bandwidth effectively available for data transmission by $2 N_{\mathrm{P}} / K=1 / 16$ (see $B_{\text {eff }}$ in Table I).
PAPR Statistics and Experiment Histogram

Probability Distribution vs. PAPR [dB]

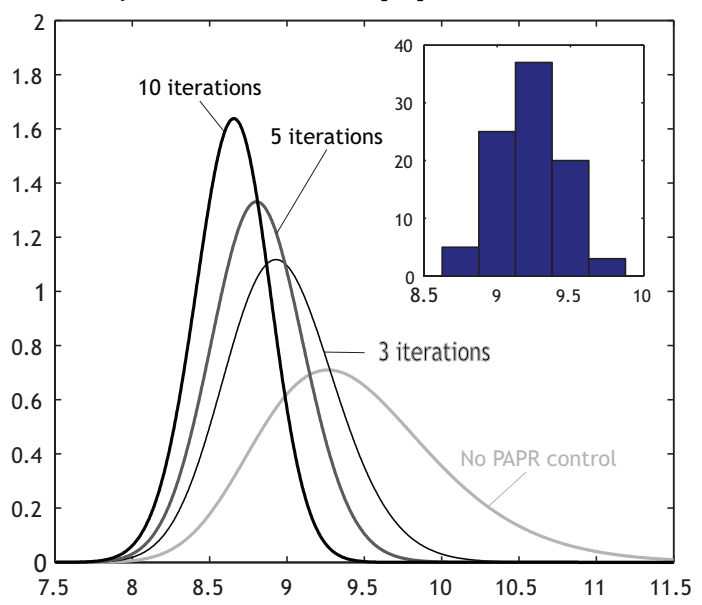

Fig. 1. Probability distribution of peak-to-average ratio (PAPR) for IFFT size of $K=4096$; shown are the distribution without PAPR control and with $N_{\text {it }}=3,5,10$ PAPR control iterations (inset is histogram of experimental data).

The resulting spectral efficiency and data rate for QPSK are

$$
\begin{aligned}
\eta & =\frac{T}{T+T_{\mathrm{CP}}} \cdot \frac{3792}{3840} \cdot \frac{3}{4} \cdot \frac{1}{2} \log _{2} 4=0.67 \mathrm{bit} / \mathrm{s} / \mathrm{Hz} \\
R & =\eta B_{\mathrm{eff}}=5.27 \mathrm{kbit} / \mathrm{s},
\end{aligned}
$$

which includes 48 null subcarriers within the active spectrum, $1 / 4$ of pilots, and error correcting coding with rate $1 / 2$. For 16-QAM the respective values are

$$
\begin{aligned}
\eta & =\frac{T}{T+T_{\mathrm{CP}}} \cdot \frac{3792}{3840} \cdot \frac{3}{4} \cdot \frac{1}{2} \log _{2} 16=1.35 \mathrm{bit} / \mathrm{s} / \mathrm{Hz} \\
R & =\eta B_{\text {eff }}=10.54 \mathrm{kbit} / \mathrm{s} .
\end{aligned}
$$

\section{PAPR Control Through Channel Coding}

\section{A. Overview PAPR}

The peak-to-average power ratio (PAPR) is commonly defined as (see [15]),

$$
\operatorname{PAPR}:=\max \left(|\tilde{x}(t)|^{2}\right) / E\left[|\tilde{x}(t)|^{2}\right] .
$$

The PAPR is important, since power amplifiers are commonly peak limited; this means that in practice it is not the average transmitted power that is constrained, but its peak value. Signals are therefore scaled before transmission to comply with a given peak power constraint. As an example, if one signal has a $3 \mathrm{~dB}$ smaller PAPR than another, it can be transmitted with $3 \mathrm{~dB}$ higher average power due to less required amplifier backoff.

While in singlecarrier transmission the peak power value is data independent (mainly defined by the pulse-shaper), for OFDM signals the peak value of each OFDM block depends on the contained data, as the IFFT outputs are effectively linear combinations of the modulated data symbols. Treating the data symbols as independent identically distributed (i.i.d.) random variables, the IFFT outputs are approximately Gaussian due to 


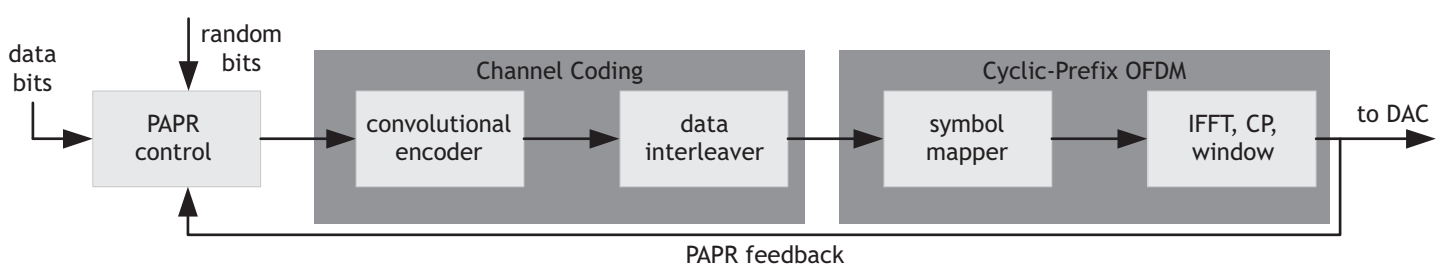

Fig. 2. The peak-to-average power ratio (PAPR) control interleaves independent random bits between the data bits before channel coding; this way $N_{\text {it }}$ seemingly independent OFDM blocks can be generated that carry the same data, the block with the lowest PAPR is transmitted.

the central limit theorem (see e.g. [16]). Therefore for an IFFT size of $K$, the PAPR of one OFDM block is distributed as the maximum magnitude of $K$ i.i.d. complex Gaussian random variables. The probability distribution function (PDF) for $K=$ 4096 is given in Fig. 1, labeled "No PAPR control". We notice that for this large value of $K$ the average PAPR of one OFDM symbol is around 9-10 dB but, more importantly, the tail of the PDF is "heavy" - this means that it decreases slowly, leaving a non-negligible probability that one OFDM block could have a PAPR value of $11-12 \mathrm{~dB}$.

Although the literature on PAPR control is extensive (see, e.g., [15] and references therein), in UWA communications the constraints on algorithmic complexity are much relaxed due to the relatively low data rates. Therefore, we focus on a simple but effective scheme suggested in [7], that is a variant of the selected mapping (SLM) approach (see [15]). The key feature is that it has no detrimental effects on bit error rate (BER) performance - but at (relatively) high complexity.

\section{B. Selected Mapping Through Channel Coding}

PAPR reduction schemes like selected mapping (SLM) make use of the randomness property of the PAPR value of OFDM blocks, i.e., if we could choose between a few (independent) OFDM blocks, say, $N_{\text {it }}$, we could surely find one that has a sufficiently low PAPR value. This is easily explained by order statistics; the PAPR value of one OFDM block is the maximum of $K$ i.i.d. random variables, and if we could choose between $N_{\text {it }}$ OFDM blocks to minimize the PAPR, the final PAPR value would be distributed as the minimum of $N_{\mathrm{it}}$ random variables, each of which is the maximum of $K$ random variables. This interlocking of maximization and minimization is quite effective in controlling the possible values. We show the corresponding PDFs in Fig. 1 for $N_{\mathrm{it}}=3,5,10$ iterations; the average PAPR reduces to 8.5$9 \mathrm{~dB}$ but, more importantly, the tails reduce significantly.

To generate $N_{\text {it }}$ independent OFDM blocks, in SLM the data bits are XORed with different pseudo-random bit sequences before mapping them to complex constellation symbols (similar as in Fig. 2). To choose the pseudo-random sequence that leads to the OFDM block with the lowest PAPR value, the transmitter has to generate $N_{\text {it }}$ possible transmit signals leading to overhead not acceptable in radio OFDM systems. A second disadvantage is that the choice of pseudorandom sequence constitutes side-information that has to be transmitted as well. While this is not a significant overhead - a pre-determined set of $N_{\mathrm{it}}=8$ pseudo-random sequences could be identified using only three bits - the possibility of transmission errors in these highly important bits would significantly impact BER performance.

Therefore the approach taken in [7] and here is that instead of XORing with a pseudo-random sequence, pseudo-random bits are interleaved within the data bits before channel coding, see Fig. 2. The application of channel coding effectively takes the place of XORing with a pseudo-random sequence, while bit errors in the pseudo-random bits do not impair recovery of the rest of the data. This works well if most encoded bits are XORed with at least one pseudo-random bit. As pointed out in [7] this is true for low-density parity check (LDPC) codes, as the generator matrix has high density. On the other hand, in convolutional codes the generator matrix has a banded structure, which means that each encoded bit has been only XORed with some preceding bits that are within the constraint length of the code.

To achieve sufficient PAPR reduction, we accordingly have to increase the number of interleaved pseudo-random bits. For the used convolutional code of constraint length 9 (polynomials 561 and 753 in octal format), we interleave a pseudorandom bit at every 20th position. This is significantly higher than needed for LDPC codes in [7], but the data rate will still not be reduced significantly: the overhead is only $5 \%$. As for the large number of pseudo-random bits we cannot exhaustively test the PAPR value for each combination, so we generate $N_{\text {it }}=10$ sets of pseudo random bits for each OFDM block and transmit the one with minimum PAPR. As an inset in Fig. 1 we show the histogram of nine bursts of $N_{\mathrm{B}}=10$ OFDM blocks (90 in total) that were transmitted (repeatedly) during the experiment. Although $N_{\text {it }}=10$ iterations are used, the average PAPR at about $9.25 \mathrm{~dB}$ is larger than theoretically predicted. This is because the $N_{\text {it }}$ iterations are not fully independent, as not all data bits are XORed with pseudorandom bits. More importantly, among 90 OFDM blocks none has a PAPR value larger than $9.8 \mathrm{~dB}$.

\section{Underwater ACOUSTiC CHANnel}

As in [10], we assume a channel consisting of few discrete arrivals with linearly time-varying delays,

$$
h(\tau ; t)=\sum_{p} A_{p} \delta\left(\tau-\left(\tau_{p}-a_{p} t\right)\right) .
$$

Each arrival is characterized by an initial delay, $\tau_{p}$, and rateof-change, $a_{p}$. Alternatively the Doppler frequency $f_{D, p}=$ $a_{p} f_{c}$ (which assumes a narrowband signal at $f_{c}$ ) could also be 

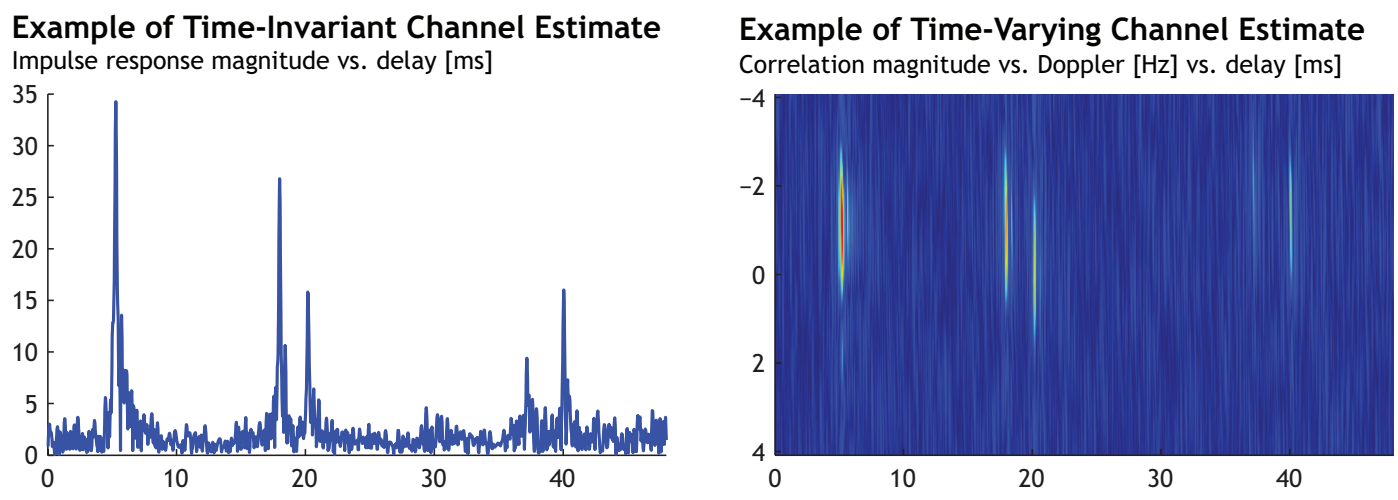

Fig. 3. Estimates of a channel response $h(\tau ; t)$ recorded at the CalCom'10 experiment; while the time-invariant estimate correlates the received signal only with different delays, the time-varying estimate additionally uses possible (wideband) Doppler shifts.

used $^{1}$. The parameters are assumed constant for the duration of one OFDM block, $T^{\prime}$, and independent across blocks.

A channel estimate is plotted in Fig. 3, where the plotted amplitudes are generated by correlating a received block with the corresponding transmitted OFDM block that has been delayed and scaled with a set of delays and Doppler frequencies $\left\{\tau_{p}, f_{D, p}\right\}$ according to (8). The delay spread, i.e., the delay difference between the first and last significant paths, is about $35 \mathrm{~ms}$ in Fig 3. Another important quantity is the Doppler spread, which is the difference between the smallest and largest Doppler frequencies. Doppler effects lead to intercarrier interference (ICI), if the Doppler spread is not much smaller than the subcarrier spacing. So, although the Doppler spread is not large (about $1.5 \mathrm{~Hz}$ ), it is significant relative to the subcarrier spacing $1 / T \approx 2 \mathrm{~Hz}$ due to the long OFDM symbol length.

\section{RECEIVED SIGNAL}

\section{A. Receiver Processing}

According to the channel model in (8), the received signal is

$$
\begin{aligned}
\tilde{y}(t) & =\tilde{x}(t) \star h(\tau ; t)+\tilde{v}(t), \\
& =\sum_{p} A_{p} \tilde{x}\left(\left(1+a_{p}\right) t-\tau_{p}\right)+\tilde{v}(t)
\end{aligned}
$$

where $\tilde{v}(t)$ is the ambient noise. The signal is downconverted, then (over-) sampled and fed into an FFT block, including a narrowband Doppler compensation $\epsilon$ (see [4] and references therein); the $m$ th FFT output of the $n$th OFDM block can be calculated as,

$$
\begin{aligned}
z_{m} & =\frac{1}{T} \int_{0}^{T} \tilde{y}\left(t+n T^{\prime}\right) e^{-j 2 \pi\left(f_{c}+\epsilon\right) t} e^{-j 2 \pi \frac{m}{T} t} d T \\
& =\sum_{k \in \mathcal{S}_{\mathrm{A}}} H[m, k] s[k]+v_{m}, m \in \mathcal{S}_{A}
\end{aligned}
$$

where we drop the index $n$ for a more compact notation. Due to the Doppler effects caused by the time-varying channel

\footnotetext{
${ }^{1}$ For narrowband signals, the channel model in (8) can be approximated by the well-known complex exponential basis expansion model (CE-BEM), see e.g. [6], [8]
}

the OFDM subcarriers loose their orthogonality, leading to ICI, where coefficient $H[m, k]$ specifies the contribution of the $k$ th subcarrier to the $m$ th FFT output. Collecting the ICI coefficients into a matrix $\mathbf{H}$, and the $z_{m}, s[k]$, and $v_{m}$ into vectors $\mathbf{z}, \mathbf{s}$, and $\mathbf{v}$, respectively, we can rewrite (11) as,

$$
\mathbf{z}=\mathbf{H} \mathbf{s}+\mathbf{v} .
$$

\section{B. Characterization of Inter-Carrier Interference}

Based on the channel model in (8), the ICI coefficients can be calculated as,

$$
\begin{aligned}
& H[m, k]=\sum_{p} \underbrace{A_{p} e^{-j 2 \pi f_{c} \tau_{p}}}_{\xi_{p}} e^{-j 2 \pi \frac{k}{T} \tau_{p}} \\
& \times \underbrace{\frac{1}{T} \int_{0}^{T} \exp \left(j 2 \pi \frac{k-m+a_{p}\left(k+f_{c} T\right)-\epsilon T}{T} t\right) d t}_{\varrho_{m, k}\left(a_{p}, \epsilon\right)},
\end{aligned}
$$

where the integral can be identified as the (inverse) Fourier transform of the rectangular receiver window implicitly used in the CP-OFDM receiver at frequency

$$
\omega_{m, k}(a, \epsilon)=\left(k-m+a\left(k+f_{c} T\right)-\epsilon T\right) / T .
$$

Creating the diagonal matrix $\boldsymbol{\Lambda}(\tau)$ with $k$ th element

$$
[\boldsymbol{\Lambda}(\tau)]_{k, k}=e^{-j 2 \pi \frac{k}{T} \tau},
$$

and the mixing matrix $\boldsymbol{\Gamma}(a, \epsilon)$ with $(m, k)$ th element

$$
[\boldsymbol{\Gamma}(a, \epsilon)]_{m, k}=\varrho_{m, k}(a, \epsilon)
$$

we can use the following vector/matrix notation

$$
\mathbf{z}=\sum_{p} \xi_{p} \boldsymbol{\Gamma}\left(a_{p}, \epsilon\right) \boldsymbol{\Lambda}\left(\tau_{p}\right) \mathbf{s}+\mathbf{v},
$$

which is equivalent to the zero-padding (ZP) OFDM formulation in [10], but the matrices depending on delays, $\tau_{p}$, and Doppler rates, $a_{p}$, have swapped order.

Based on the relationship between the discrete paths and the ICI in (17), sparse channel estimation can be applied to first estimate the channel parameters and then to reconstruct the channel matrix as in [10]. We consider two reconstruction 
algorithms: Orthogonal Matching Pursuit (OMP) [13] and Basis Pursuit (BP) [14] (see [10] for implementation details).

For comparison we include the case where the channel is assumed to be time-invariant, i.e., all $a_{p}=0$ and $\mathbf{H}$ is a diagonal matrix. In this case a conventional least-squares (LS) estimator, which does not take advantage of the channel sparsity, can also be used. When considering the time-varying channel model, we indicate this as "OMP (ICI)" and "BP (ICI)" .

\section{Multi-Phone Combining and Soft-Information}

Since most underwater acoustic receivers are arrays, the output of multiple receivers can be combined to improve BER performance. We index the FFT outputs of the $r$ th receiver as,

$$
\mathbf{z}_{r}=\mathbf{H}_{r} \mathbf{S}+\mathbf{v}_{r} .
$$

If there are $N_{\mathrm{R}}$ receivers, the (linear) relationship to the unknown data vector $\mathbf{s}$ is,

$$
\left[\begin{array}{c}
\mathbf{z}_{1} \\
\vdots \\
\mathbf{z}_{N_{\mathrm{R}}}
\end{array}\right]=\left[\begin{array}{c}
\mathbf{H}_{1} \\
\vdots \\
\mathbf{H}_{N_{\mathrm{R}}}
\end{array}\right] \mathbf{s}+\left[\begin{array}{c}
\mathbf{v}_{1} \\
\vdots \\
\mathbf{v}_{N_{\mathrm{R}}}
\end{array}\right] .
$$

To demodulate the data we use the (linear) minimum meansquare error (MMSE) equalizer (see, e.g., [17]),

$$
\hat{\mathbf{s}}=\left(\sum_{r=1}^{N_{\mathrm{R}}} \frac{\mathbf{H}_{r}^{H} \mathbf{H}_{r}}{N_{0}^{(r)}}+\mathbf{I}\right)^{-1} \sum_{r=1}^{N_{\mathrm{R}}} \frac{\mathbf{H}_{r}^{H} \mathbf{z}_{r}}{N_{0}^{(r)}},
$$

where we assumed that the noise $\mathbf{v}_{r}$ has covariance $N_{0}^{(r)} \mathbf{I}$.

To decode the convolutional code, a soft-input Viterbi decoder is used, where soft-input means that we need to provide a measure of reliability of each bit estimate, typically the loglikelihood. Although the exact log-likelihood of the symbol estimates in (20) cannot be determined easily, approximating the estimates as Gaussian with matched first and second moments is a reasonable approach, as suggested in [18]. Assuming,

$$
\hat{s}[k]=\mu_{k} s[k]+w_{k},
$$

where $\mu_{k}$ is a scaling factor and $w_{k}$ is zero-mean complex Gaussian with power $\sigma_{k}^{2}$ that accounts for filtered noise, as well as un-equalized ICI from the $s\left[k^{\prime}\right], k^{\prime} \neq k$. To determine these parameters we condition on only one symbol at a time

$$
\begin{aligned}
\mu_{k} & =E[\hat{s}[k] \mid s[k]] / s[k] \\
& =\mathbf{e}_{k}^{H}\left[\left(\sum_{r=1}^{N_{\mathrm{R}}} \frac{\mathbf{H}_{r}^{H} \mathbf{H}_{r}}{N_{0}^{(r)}}+\mathbf{I}\right)^{-1} \sum_{r=1}^{N_{\mathrm{R}}} \frac{\mathbf{H}_{r}^{H} \mathbf{H}_{r}}{N_{0}^{(r)}}\right] \mathbf{e}_{k}
\end{aligned}
$$

where $\mathbf{e}_{k}$ is the $k$ th basis vector, basically selecting the $k$ th diagonal element of the matrix between brackets. We term the diagonal elements $D_{k}$, accordingly

$$
\mu_{k}=D_{k} .
$$

The second moment is

$$
\sigma_{k}^{2}=\operatorname{Cov}[\hat{s}[k] \mid s[k]]=D_{k}\left(1-D_{k}\right),
$$

Initial CalCom'10 Results for QPSK @5.27 kbit/s
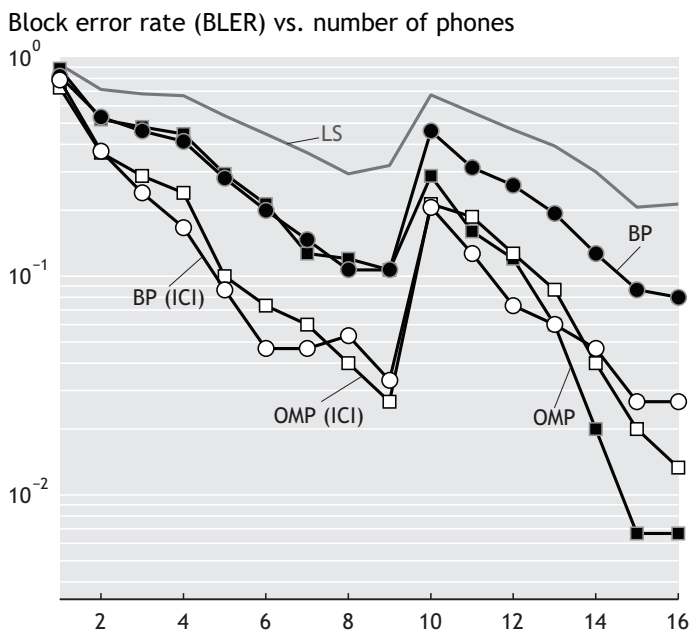

Fig. 4. Recorded performance showing unusual performance loss when including receive element ten.

where we refer to [18] for the detailed derivation.

Finally, based on the Gaussian model, we generate a $\log$ likelihood ratio (LLR) for each bit decision related to symbol $s[k]$. To this end assume that the symbol $s[k]$ is drawn from an $M$-ary constellation:

$$
s[k] \in\left\{\alpha_{m}\right\}_{m=1}^{M}
$$

Each symbol is associated with $\log _{2} M$ bits which we refer to as $b[i, k] \in\{0,1\}, i=1, \ldots, \log _{2} M$. Now the LLR of bit $b[i, k]$ is,

$$
\begin{aligned}
\operatorname{LLR}[i, k] & =\ln \frac{P(b[i, k]=1 \mid \hat{s}[k])}{P(b[i, k]=0 \mid \hat{s}[k])} \\
& =\ln \frac{\sum_{m=1}^{M} \delta[i, m] e^{-d_{m}^{2}[k]}}{\sum_{m=1}^{M}(1-\delta[i, m]) e^{-d_{m}^{2}[k]}},
\end{aligned}
$$

where the normalized distance of each symbol $\alpha_{m}$ from observation $\hat{s}[k]$ is

$$
d_{m}^{2}[k]=\frac{\left|\hat{s}[k]-\mu_{k} \alpha_{m}\right|^{2}}{\sigma_{k}^{2}},
$$

and the bit index $\delta[i, m]$ is one if the $i$ th bit of symbol $\alpha_{m}$ is one

$$
\left(s[k]=\alpha_{m} \wedge \delta[i, m]=1\right) \Rightarrow b[i, k]=1 .
$$

\section{Colored Noise Spectrum And Interference}

\section{A. Observation in Experimental Data}

When decoding the Calcom'10 experimental data as described in Section V, the results showed an unexpected behavior, depicted in Fig. 4. The plot shows block error rate (BLER), which is the fraction of OFDM blocks that had one or more bit errors after decoding the error correcting code. It is plotted against an increasing number of phones, which corresponds to increasing $N_{\mathrm{R}}$ in (19) until all sixteen receive phones are combined. 
Noise Spectrum Estimate at Phone 10

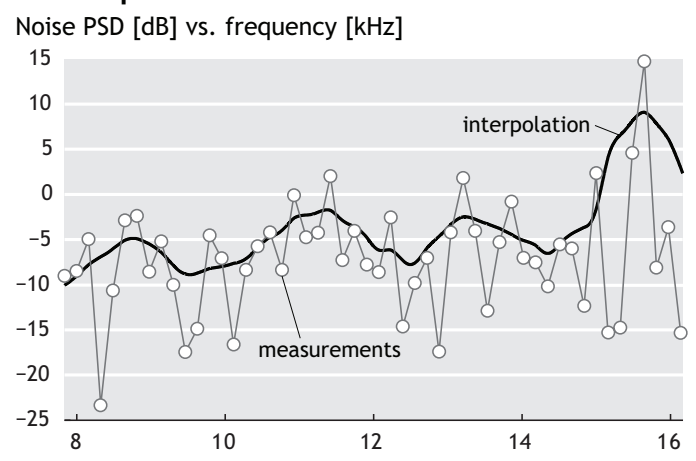

Noise Spectrum Estimate at Phone 14

Noise PSD [dB] vs. frequency [kHz]

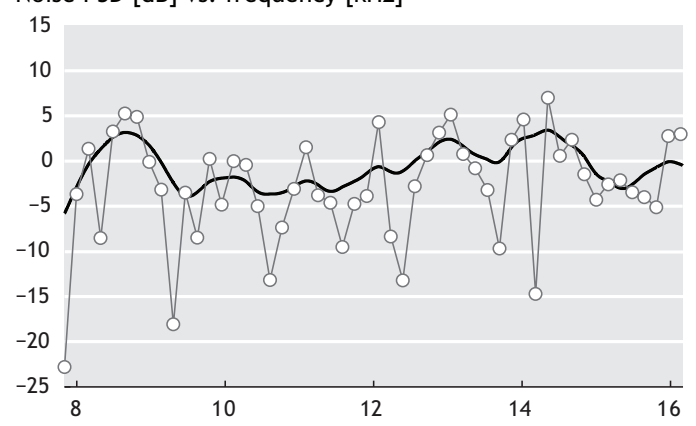

Fig. 5. Examples of noise observations on null subcarriers and noise spectrum estimates based on interpolation; in phone 10 a strong noise spike between $15-16 \mathrm{kHz}$ seems to be the cause of the erratic performance in Fig. 4.

Theoretically the BLER should be a strictly decreasing quantity with respect to $N_{\mathrm{R}}$. This assumes perfect knowledge of the channel/ICI coefficients $\mathbf{H}_{r}$ and the noise levels $N_{0}^{(r)}$. Although in practice these quantities are only known approximately, the sharp increase in BLER when including the tenth phone is unusual. As the phenomena was consistent across all channel estimation schemes, the assumption was that a significantly colored noise spectrum might be the cause for the erratic behavior. As a remedy noise whitening as proposed in [11] was considered.

\section{B. Noise Spectrum Estimation Using Null Subcarriers and Interpolation}

MMSE multi-phone combining, as described in Section $\mathrm{V}-\mathrm{C}$, assumes that the noise is white, i.e.,

$$
\operatorname{Cov}\left[\mathbf{v}_{r}\right]=N_{0}^{(r)} \mathbf{I} \text {. }
$$

The noise power at the $r$ th receiver is commonly estimated as (see [4] and references therein)

$$
\hat{N}_{0}^{(r)}=\frac{1}{\left|\mathcal{S}_{N}\right|} \sum_{m \in \mathcal{S}_{N}}\left|z_{r, m}\right|^{2},
$$

where $z_{r, m}$ refers to the elements of $\mathbf{z}_{r}$.

Instead, as in [11], we will allow the noise to be correlated in the time domain, leading to a varying power spectral density (PSD) across the OFDM subcarriers,

$$
\operatorname{Cov}\left[\mathbf{v}_{r}\right]=\mathbf{D}_{r} .
$$

\section{Results for QPSK Including Noise Spectrum}

Block error rate (BLER) vs. number of phones

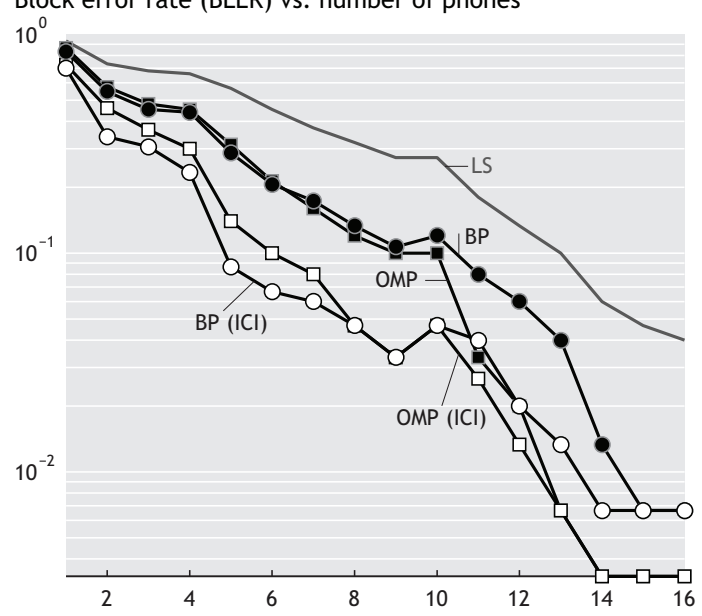

Fig. 6. Performance including estimated noise PSD.

Accordingly $\mathbf{D}_{r}$ is a diagonal matrix that can vary per phone; the coefficients can be estimated using the observations of noise on the 48 null subcarriers that are evenly spaced within the used frequency band.

Unlike [11], we do not use any specific model to characterize the noise PSD. Instead, the observations of the noise power are simply averaged using a length fifteen triangular window to avoid negative biasing, as explained in [11], and increase smoothness. Then the coefficients corresponding to active subcarriers are estimated using interpolation in the logarithmic domain. The estimator in (32) would in this sense correspond to using a length $K$ rectangular window.

Null subcarrier measurements and estimated noise PSDs are plotted in Fig. 5; the noise estimates are normalized such that $\hat{N}_{0}^{(r)}$ corresponds to $0 \mathrm{~dB}$. The noise level generally varies by up to $\pm 5 \mathrm{~dB}$, which is significant, but, more importantly, for phone 10 we see a noise spike between 15-16 kHz. As shown in Fig 6, the performance can be improved by including the colored noise estimates in MMSE multi-phone combining.

\section{Biased Symbol Estimates Due to Channel ESTIMATION ERRORS}

\section{A. Sample Mean of Symbol Estimates}

To check if the symbol estimates in (21) are unbiased in practice, we re-normalized them to

$$
\hat{s}_{\mu}[k]=\hat{s}[k] / \mu_{k}=s[k]+w_{k} / \mu_{k},
$$

which is also a Gaussian model, with scaled mean and variance relative to (21). In fact, for the channel estimators that assume the channel to be time-invariant (diagonal $\mathbf{H}_{r}$ ), the MMSE equalizer reduces to maximum-ratio combining (MRC),

$$
\hat{s}_{\mu}[k]=\frac{\sum_{r=1}^{N_{\mathrm{R}}} H_{r}^{*}[k, k] z_{r, k}}{\sum_{r=1}^{N_{\mathrm{R}}}\left|H_{r}[k, k]\right|^{2}},
$$

which is also the LS solution to (19). 
Mean and Spread of LS Symbol Estimates Quadrature vs. in-phase component

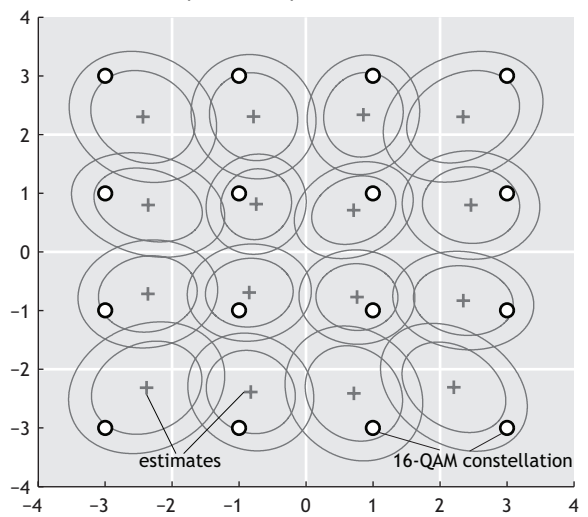

Mean and Spread of OMP Symbol Estimates Quadrature vs. in-phase component

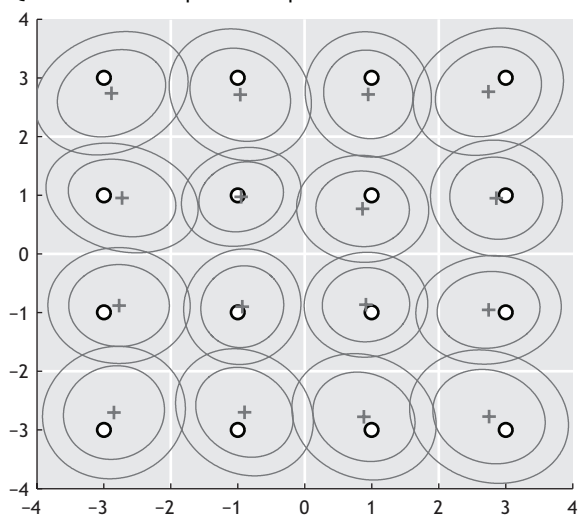

Mean and Spread of BP Symbol Estimates Quadrature vs. in-phase component

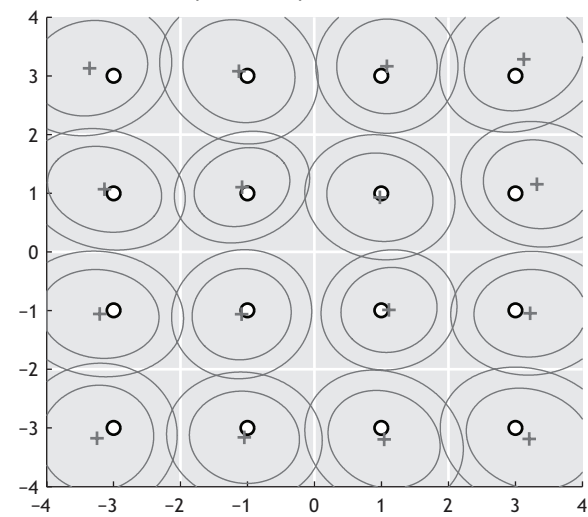

Mean and Spread of OMP (ICI) Symbol Estimates Quadrature vs. in-phase component

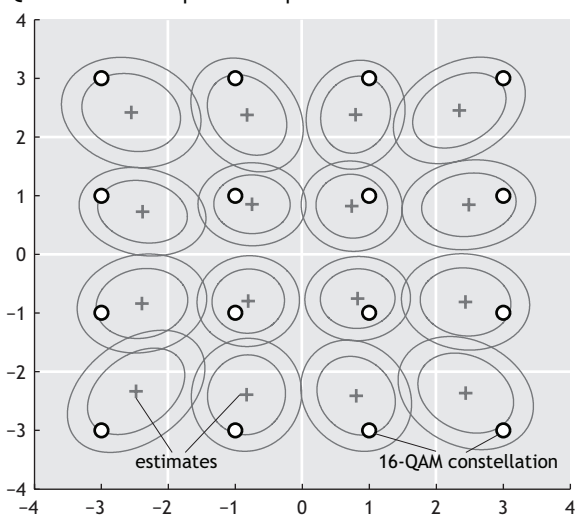

Mean and Spread of BP (ICI) Symbol Estimates Quadrature vs. in-phase component

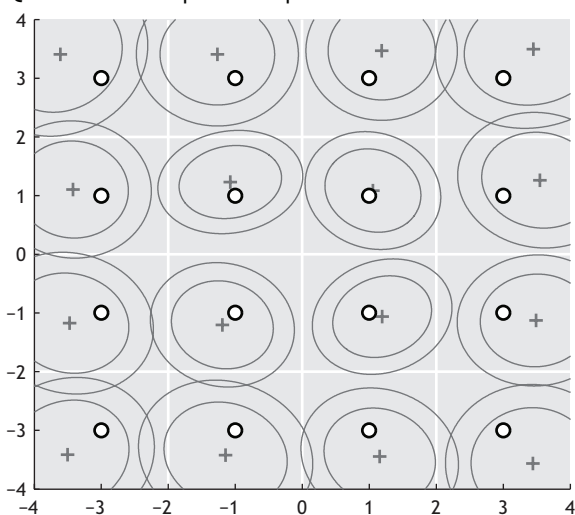

Fig. 7. Symbol estimates are significantly biased; example of one OFDM block after multi-phone combining; circles are actual constellation points, crosses mark mean of symbol estimates, while ellipses correspond to one and two standard deviations; grey quadrants correspond to hard decision boundaries.

We experimentally measure the complex mean and variance of $\hat{s}_{\mu}[k]$ given that $s[k]=\alpha_{m}$. For this, we group all $s[k]$ that take the same constellation point,

$$
\mathcal{S}_{m}=\left\{k \mid s[k]=\alpha_{m}\right\} .
$$

Now we use the sample mean based on one OFDM block,

$$
\bar{s}_{m}=\frac{1}{\left|\mathcal{S}_{m}\right|} \sum_{k \in \mathcal{S}_{m}} \hat{s}_{\mu}[k] \approx E\left[\hat{s}_{\mu}[k] \mid s[k]=\alpha_{m}\right] .
$$

As the model in (21) has exactly derived first and second moments, this symbol estimate should be unbiased.

We measure the sample mean and the spread (covariance matrix treating the complex number as a two-dimensional vector), which are plotted in Fig. 7 for 16-QAM data and all considered channel estimation schemes, based on full multi-phone combining. Clearly not all symbol estimates are unbiased, especially for the conventional LS channel estimator, as well as for both OMP and BP when estimating the ICI pattern.

For BP it is known that the channel estimates tend to be biased towards zero, for which an additional debiasing stage is sometimes suggested, e.g., in [19]. We do not use debiasing for BP, therefore the smaller channel estimates lead in turn to enlarged symbol estimates as observed for BP in Fig. 7. For OMP and, especially, LS channel estimators, biased estimates are unusual. Additionally, the difference in OMP and BP between assuming the channel as time-invariant or time-varying (estimate ICI) indicates that this effect may be attributed to other causes besides channel estimation.

\section{B. Unbiased Maximum-Ratio Combining}

To explain the observed effect, we consider the following example based on a time-invariant channel for simplicity. Assume the received sample was simply

$$
z_{r, k}=H_{r}[k, k] s[k]+v_{r, k},
$$

where for simplicity we assume a constant noise level $N_{0}$ across all receivers $r=1, \ldots, N_{\mathrm{R}}$. Now applying MRC as in (35), but with noisy channel estimates

$$
\hat{H}_{r}[k, k]=H_{r}[k, k]+w_{r, k},
$$

of variance $\sigma_{h}^{2}$, the symbol estimate becomes,

$$
\hat{s}_{\mu}[k]=\frac{\sum_{r=1}^{N_{\mathrm{R}}} \hat{H}_{r}^{*}[k, k] H_{r}[k, k] s[k]+\hat{H}_{r}^{*}[k, k] v_{r, k}}{\sum_{r=1}^{N_{\mathrm{R}}}\left|H_{r}[k, k]\right|^{2}+2 \Re\left\{H_{r}[k, k] w_{r, k}^{*}\right\}+\left|w_{r, k}\right|^{2}} .
$$




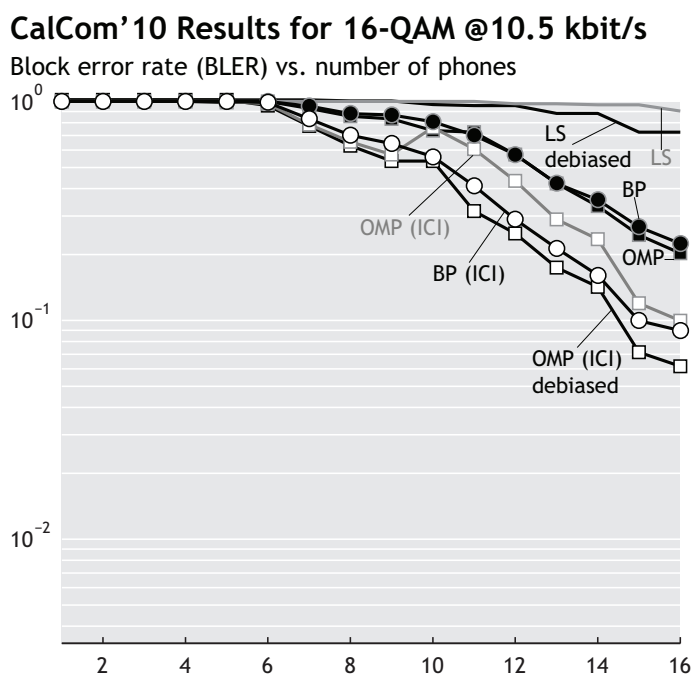

Fig. 8. Performance for 16-QAM, including debiasing for OMP and LS.

For large number of phones, $N_{\mathrm{R}} \rightarrow \infty$, this quantity will converge to

$$
\hat{s}_{\mu}[k]=\nu_{k} s[k]+\tilde{v}_{k}
$$

with Gaussian noise $\tilde{v}_{k}$ and scale

$$
\nu_{k}=\frac{\frac{1}{N_{\mathrm{R}}} \sum_{r=1}^{N_{\mathrm{R}}}\left|H_{r}[k, k]\right|^{2}}{\frac{1}{N_{\mathrm{R}}} \sum_{r=1}^{N_{\mathrm{R}}}\left|H_{r}[k, k]\right|^{2}+\sigma_{h}^{2}},
$$

which depends on the channel estimation error.

This seems to fit the observations in several ways: i) the symbol estimates are scaled down for LS and OMP channel estimators, while for BP the implicit biasing of the channel estimates outweighs this effect; this is most likely also a reason for BP outperforming OMP in [10] when estimating ICI. ii) The channel estimation error will be smaller when approximating the channel matrix $\mathbf{H}_{r}$ as diagonal, therefore these estimators are less biased than the respective versions that model ICI; this is further confirmed by results using an increased (double) amount of pilots - the bias decreases significantly in this case.

Finally, in Fig. 8 we show performance results using an ad-hoc debiasing scheme for OMP and LS. The channel estimation error is assumed proportional to the (also estimated) noise level $N_{0}^{(r)}$. The performance increase in both OMP and LS is significant, but a more sophisticated debiasing strategy will remain for future work.

\section{CONCLUSION}

We presented experimental results collected at a recent seatrial. After outlining the employed signal design and receiver processing, we focused on two unexpected effects that were observed in the results. The first is colored noise caused by unaccounted intercarrier interference and a narrowband interference source. The second is biased symbol estimates due to noisy channel estimates used in multi-phone combining. We study both effects and suggest simple remedies to recover losses in terms of decoding performance. Both effects warrant further investigation.

\section{ACKNOWLEDGEMENT}

The work of Christian R. Berger and José M. F. Moura was partially supported by ONR grant \# N000141110112. The work of João Gomes was partially funded by "Fundação para a Ciência e a Tecnologia", the CMU/Portugal Program managed by ICTI, and ISR/IST plurianual funding. The authors also thank CINTAL/Universidade do Algarve for conducting most of the activities in the CalCom'10 sea trial.

\section{REFERENCES}

[1] T. H. Eggen, A. B. Baggeroer, and J. C. Preisig, "Communication over Doppler spread channels. Part I: Channel and receiver presentation," IEEE J. Ocean. Eng., vol. 25, no. 1, pp. 62-71, Jan. 2000.

[2] D. B. Kilfoyle and A. B. Baggeroer, "The state of the art in underwater acoustic telemetry," IEEE J. Ocean. Eng., vol. 25, no. 1, pp. 4-27, Jan. 2000.

[3] M. Stojanovic, "Low complexity OFDM detector for underwater channels," in Proc. of MTS/IEEE OCEANS Conf., Boston, MA, Sept. 18-21, 2006.

[4] B. Li, S. Zhou, M. Stojanovic, L. Freitag, and P. Willett, "Multicarrier communication over underwater acoustic channels with nonuniform Doppler shifts," IEEE J. Ocean. Eng., vol. 33, no. 2, pp. 198-209, Apr. 2008.

[5] T. Kang and R. A. Iltis, "Iterative carrier frequency offset and channel estimation for underwater acoustic OFDM systems," IEEE J. Select. Areas Commun., vol. 26, no. 9, pp. 1650-1661, Dec. 2008.

[6] G. Leus and P. A. van Walree, "Multiband OFDM for covert acoustic communications," IEEE J. Select. Areas Commun., vol. 26, no. 9, pp. 1662-1673, Dec. 2008.

[7] J. Huang, S. Zhou, and P. Willett, "Nonbinary LDPC coding for multicarrier underwater acoustic communication," IEEE J. Select. Areas Commun., vol. 26, no. 9, pp. 1684-1696, Dec. 2008.

[8] S.-J. Hwang and P. Schniter, "Efficient multicarrier communication for highly spread underwater acoustic channels," IEEE J. Select. Areas Commun., vol. 26, no. 9, pp. 1674-1683, Dec. 2008.

[9] K. Tu, D. Fertonani, T. M. Duman, and P. Hursky, "Mitigation of intercarrier interference in OFDM systems over underwater acoustic channels," in Proc. of MTS/IEEE OCEANS Conf., Bremen, Germany, May 2009.

[10] C. R. Berger, S. Zhou, J. Preisig, and P. Willett, "Sparse channel estimation for multicarrier underwater acoustic communication: From subspace methods to compressed sensing," IEEE Trans. Signal Processing, vol. 58, no. 3, pp. 1708-1721, Mar. 2010.

[11] C. R. Berger, W. Chen, S. Zhou, and J. Huang, "A simple and effective noise whitening method for underwater acoustic orthogonal frequency division multiplexing," J. Acoust. Soc. Am., vol. 127, no. 4, pp. 23582367, Apr. 2010.

[12] W. Li and J. C. Preisig, "Estimation of rapidly time-varying sparse channels," IEEE J. Ocean. Eng., vol. 32, no. 4, pp. 927-939, Oct. 2007.

[13] S. Mallat and Z. Zhang, "Matching pursuits with time-frequency dictionaries," IEEE Trans. Signal Processing, vol. 41, no. 12, pp. 3397-3415, Dec. 1993.

[14] S. S. Chen, D. L. Donoho, and M. A. Saunders, "Atomic decomposition by basis pursuit," SIAM J. Scientific Computing, vol. 20, no. 1, pp. 3361, 1999.

[15] S. Litsyn, Peak Power Control in Multicarrier Communications, 1st ed. Cambridge University Press, Jan. 2007.

[16] A. Papoulis and S. U. Pillai, Probability, Random Variables and Stochastic Processes. New York: McGraw-Hill, 2002.

[17] J. G. Proakis, Digital Communications, 4th ed. New York: McGrawHill, 2001.

[18] M. Tuechler, A. C. Singer, and R. Koetter, "Minimum mean square error equalization using a priori information," IEEE Trans. Signal Processing, vol. 50, no. 3, pp. 673-683, Mar. 2002.

[19] S. J. Wright, R. D. Nowak, and M. A. T. Figueiredo, "Sparse reconstruction by separable approximation," IEEE Trans. Signal Processing, vol. 57, no. 7, pp. 2479-2493, Jul. 2009. 